Full Length Article

\title{
Damage in a cast AlSi12Ni alloy: In situ tomography, 2D and 3D image correlation
}

\author{
A. Tireira ${ }^{\mathrm{a}}$, M. Bornert ${ }^{\mathrm{b}}$, G. Requena ${ }^{\mathrm{c}}$, E. Maire ${ }^{\mathrm{d}}$, S. Sao Jao ${ }^{\mathrm{a}}$, A. Borbely ${ }^{\mathrm{a}}$, H. Klocker ${ }^{\mathrm{a}, *}$ \\ ${ }^{a}$ Mines Saint-Etienne, Univ Lyon, CNRS, UMR 5307 LGF, Centre SMS, F - 42023 Saint-Etienne France \\ ${ }^{\mathrm{b}}$ Laboratoire Navier, Ecole des Ponts ParisTech, CNRS UMR8205, IFSTTAR, Université Paris Est, 6 / 8 avenue Blaise Pascal, Champs sur Marne, 77455 Marne La \\ Vallée, France \\ ' German Aerospace Centre, Institute of Materials Research, Linder Höhe 51147, Cologne, Germany \\ d INSA-Lyon, MATEIS, Université de Lyon, CNRS UMR5510, F-69621 Villeurbanne, France
}

\section{A R T I C L E I N F O}

\section{Keywords:}

Aluminum alloys

In situ micro-tomography

Damage

3D image correlation

\begin{abstract}
A B S T R A C T
Damage evolution during tensile straining of an AlSi12Ni alloy has been analyzed in situ at synchrotron source using microtomography and in the scanning electron microscope by surface imaging. It is shown that damage development in the analyzed alloy presenting an interconnected network of intermetallic phases is completely different from damage progression previously observed in materials with disperse distribution of particles. In the present material, which is typical for most eutectic structures, damage is dominated by the rupture of the brittle intermetallic phase while void growth is limited by a shielding effect of the intermetallic particles encasing the void. Primary voids exhibit a size close to the thickness of branches of the intermetallic phase. Final failure occurs by void coalescence, but without the formation of secondary voids. Damage analysis from tomographic scans was only possible by applying 3D image correlation to successive reconstructions, which thanks to its sub-voxel resolution, could satisfactorily detect the volume fraction of small-voids inaccessible by conventional thresholding. The presence of many small voids issued from the breakage of the intermetallic phase also was confirmed by scanning electron microscopy imaging performed at higher resolution.
\end{abstract}

\section{Introduction}

Cast Al-Si alloys are widely used in the automotive industry for cylinder heads, pistons and engine crankcases due to their excellent castability, lower weight and better heat conductivity compared to cast iron. Standardized commercial cast alloys with Si-content in the range of 7 to 23 wt.\% exhibit typical hypoeutectic, eutectic and hypereutectic microstructures [1] and contain an $\alpha$-Al matrix and several rigid phases. For near-eutectic concentrations, an almost pure Si phase solidifies in form of branched interconnected plates [2-4]. Furthermore, three dimensional (3D) interconnected networks of brittle intermetallic (IM) phases (e.g. $\mathrm{Al}_{9} \mathrm{FeNi}$ and $\mathrm{Al}_{15} \mathrm{Si}_{2}(\mathrm{FeMn})_{3}$ ) are also present, which are usually connected to the eutectic and the Si phase forming a rigid and highly interpenetrating structure [5]. Heat treatment induced particle spherodization was analyzed thoroughly in several works [2-7], while the true relation between overall strength and particle connectivity could be only estimated after revealing the 3D structure of different phases by Synchrotron Micro-Tomography (SMCT) [8]. Due to its brittleness however, the 3D network of IM phases and Si can easily break during thermo-mechanical processing, leading to damage accumulation and crack formation [2-7]. Therefore, understanding the damage behavior of the material is important in practice. Scientific literature on the subject is relatively rare, most of the modeling work being done on materials with dispersed second phases [9-11]. In such materials damage has frequently been observed to take place in three distinct steps: nucleation, growth and coalescence of primary voids. Primary voids nucleate by decohesion of particle-matrix interfaces or by cracking of second-phase particles [11]. Then these voids grow until the strain localizes in the ligament between them. The latter is called void coalescence and leads to accelerated growth of the primary voids, secondary void formation and final failure [11]. It is therefore important to know if damage development in the AlSi12Ni alloy, presenting an interconnected network of second phases follows the same path or nor not as in microstructures with disperse phases. For example, Huber et al. [12] described damage development in an AlSi12Ni alloy after spherodization treatments with a modified Gurson model taking into account void growth. However, it isn't evident how the mechanisms of void nucleation, growth and coalescence interact, which needs advanced techniques for characterization. In this respect synchrotron based in situ techniques are very well suited as they have reached acceptable spatial (of the order of $\mu \mathrm{m}$ ) $[13,14]$ and

\footnotetext{
* Corresponding author.

E-mail address: klocker@emse.fr (H. Klocker).
} 
a

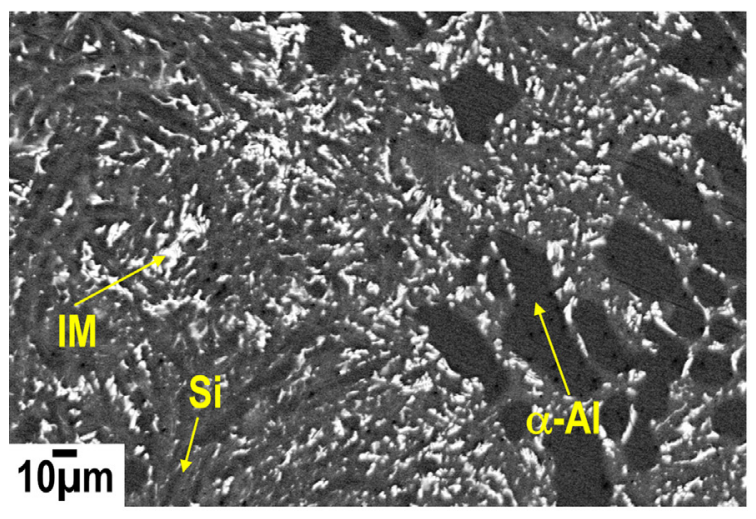

b

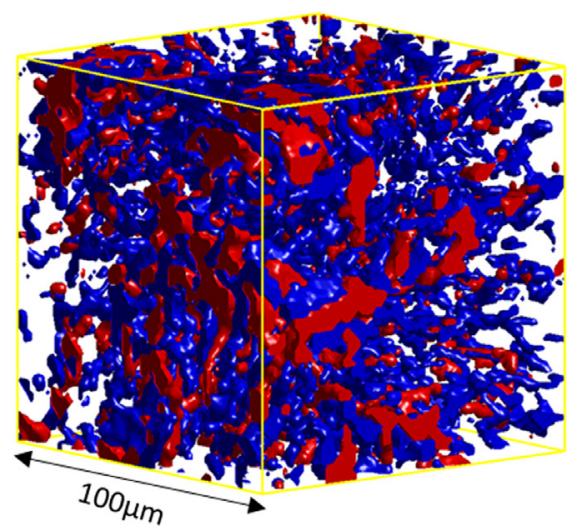

Fig. 1. Microstructure of the cast AlSi12Ni alloy. (a) SEM micrograph with IM and Si-particles embedded in an $\alpha$-Al matrix, (b) 3D SMCT reconstruction obtained at ID19 ESRF depicting the highly interconnected Si (blue) and IM-particles (red).

Table 1

Chemical composition of the studied AlSi12Ni alloy (in wt.\%).

\begin{tabular}{lllll}
\hline $\mathrm{Si}$ & $\mathrm{Ni}$ & $\mathrm{Fe}$ & $\mathrm{Mn}$ & $\mathrm{Al}$ \\
\hline 11.92 & 1.22 & 0.69 & 0.27 & Bal. \\
\hline
\end{tabular}

time resolutions (of the order of seconds) [13-16] required for describing both the complex 3D microstructure and evolution of damage. To enhance spatial resolution, image correlation has been applied to succeeding 3D tomographic images and a similar in situ tensile test has been performed in the scanning electron microscope (SEM), which provided complementary information on damage at a lower length scale. The main question addressed in this work concerns the interplay between different basic damage mechanisms in determining the final rupture of a tensile deformed AlSi12Ni specimen containing a 3D network of interconnected particles.

\section{Experimental}

\subsection{Material and microstructure}

An AlSi12Ni alloy with chemical composition as given in Table 1 was produced by squeeze casting. The material was investigated at room temperature after a spheroidization heat treatment and artificial aging [15] (Fig. 1a). The microstructure of the alloy consists of an $\alpha$-Al matrix and $\sim 8$ vol.\% of intermetallic phases $\left(\mathrm{Al}_{9} \mathrm{FeNi}\right.$ and $\left.\mathrm{Al}_{15} \mathrm{Si}_{2}(\mathrm{FeMn})_{3}\right)$, with a hardness of $\sim 8$ and $11 \mathrm{GPa}$, and $\sim 12$ vol.\% eutectic Si $[6,8]$. The 3D structure of the different phases was first characterized by highresolution SMCT at the ID19 beamline of the European Synchrotron Radiation Facility (ESRF) [5]. The reconstructed volume had a final size of $20,48^{3}$ voxels $\left(614^{3} \mu \mathrm{m}^{3}\right)$ with a voxel size of $0.3 \mu \mathrm{m}^{3}$. Fig. $1 \mathrm{~b}$ depicts a $3 \mathrm{D}$ network of complex and highly interpenetrating intermetallic and Si-particles.

\subsection{In situ characterization during tensile loading}

Particle break-up and damage progression during tensile loading were first characterized over an area of about $3 \times 3 \mathrm{~mm}^{2}$ by $2 \mathrm{D}$ in situ observations on $5 \mathrm{~mm}$-gage length specimens in the SEM. Fig. 2a shows the corresponding stress-strain curve. The engineering strain corresponds to the total sample elongation between dashed lines $\left(\varepsilon=\left(L-L_{0}\right) / L_{0}\right)$, see Fig. 2a) [15]. The in situ test in the SEM was done at a strain rate of $10^{-3} \mathrm{~s}^{-1}$. The test was not interrupted for image acquisition, which took 10 s. A similar tensile test coupled with SMCT (voxel size $\sim(1.4 \mu \mathrm{m})^{3}$ ) was performed in situ at the ID15 beamline of the ESRF on a sample with a cross section of $1 \times 1 \mathrm{~mm}^{2}$, a gage length of $30 \mathrm{~mm}$ and an ellipsoidal notch at the center of one lateral face (Fig. 2b). The remote strain corresponds to the total sample elongation, measured by an LVDT. The in situ deformation was done continuously at a strain rate of about $10^{-4} \mathrm{~s}^{-1}$, which was slow enough compared to the duration of $20 \mathrm{~s}$ of a whole tomographic scan ( 850 projections were taken during $180^{\circ}$ rotation of the sample using an exposure time of $18 \mathrm{~ms}$ per projection). The total reconstructed volume is about $1.4 \times 1.4 \times 1.1 \mathrm{~mm}^{3} .28$ scans were acquired during the tensile test performed with a dedicated load frame [16]. The locations of the analyzed states are indicated by square symbols on the stress-strain curve shown in Fig. 2b. For both tests, the load was measured by a standard load cell. The engineering stress (measured load/gage initial area) was considered. Compared to the stressstrain curve of the sample deformed in the SEM (Fig. 2a) both the tensile strength and the ductility are lower, which is a consequence of the higher stress concentration factor at the notch (4.75 and 3 for tests at ESRF and in the SEM, respectively). The stress concentration around the notch allows visualizing damage progression in the selected volume from the first events of IM break-up until final failure.

\section{3D image correlation applied to damage analysis}

In the late 1980s, Kimura and Takamori [17] and Adrian, [18] established the principles of particle image velocimetry in fluids. Since then, these techniques were widely adapted to the determination of displacement fields on solid surfaces [18-22] and have been later extended to the determination of three-dimensional movements of volume elements [23-25]. Two three-dimensional images representing a sample in a reference state and a deformed state are mapped at multiple points in a three-dimensional regular virtual grid arranged inside the volume. The movement of each point is estimated by following the movements of the gray levels of its neighborhood between two states under the assumption of material transport of the latter. Be $\mathbf{X}$ and $\mathbf{x}$ the coordinates in voxels of the same material point in the initial and final images, respectively. The material transformation linking the two configurations is written as:

$\mathbf{x}(\mathbf{X})=\mathbf{X}+\mathbf{u}(\mathbf{X})$

For a volume $\mathrm{D}$ surrounding $\mathbf{X}_{\mathbf{0}}$ in the reference image the displacement $\mathbf{u}(\mathbf{X})$ is approximated by its first-order development

$\mathbf{u}(\mathbf{X}) \approx \mathbf{u}\left(\mathbf{X}_{0}\right)+\frac{\partial \mathbf{u}}{\partial \mathbf{X}}\left(\mathbf{X}_{0}\right)\left(\mathbf{X}-\mathbf{X}_{0}\right)$

which corresponds to a rigid body movement and a homogeneous deformation throughout D

$\mathbf{x}(\mathbf{X})=\mathbf{X}+\mathbf{u}\left(\mathbf{X}_{0}\right)+\frac{\partial \mathbf{u}}{\partial \mathbf{X}}\left(\mathbf{X}_{0}\right)\left(\mathbf{X}-\mathbf{X}_{0}\right)$ 

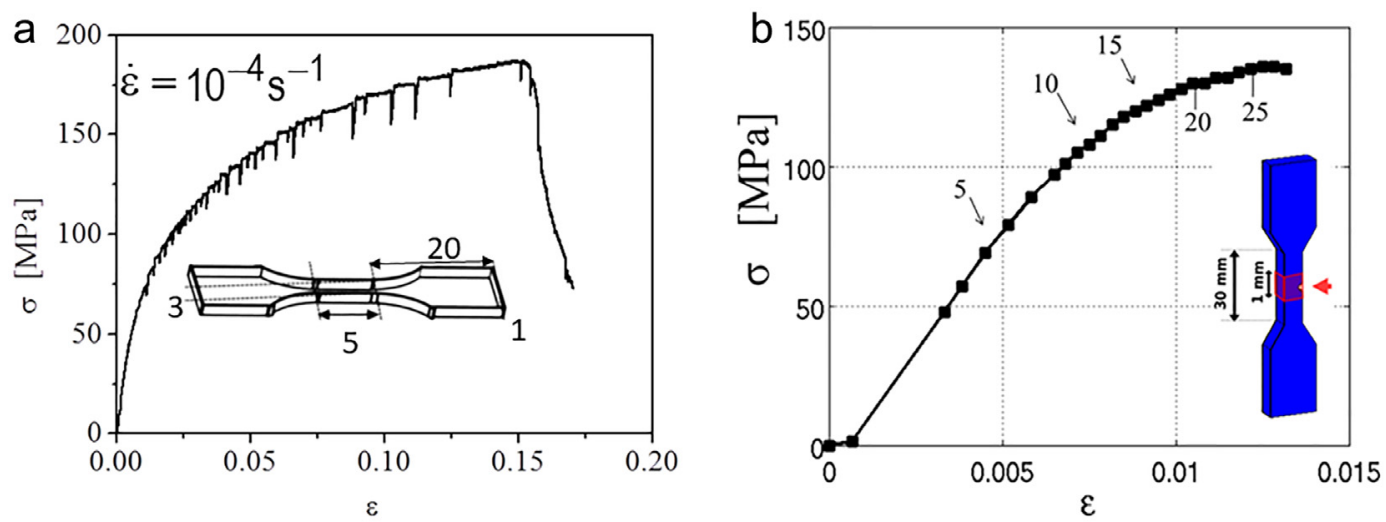

Fig. 2. Typical stress strain curves and sample geometries used for in situ tensile tests in the SEM (a) and SMCT (b).

The displacement of the center of the sub-volume $\mathrm{D}, \mathbf{u}\left(\mathbf{X}_{0}\right)$ gives the intensity of the rigid body translation, depending on 3 parameters $\left(\mathrm{u}_{1}, \mathrm{u}_{2}, \mathrm{u}_{3}\right)$. The expression of the local gradient $[\partial \mathbf{u} / \partial \mathbf{X}]\left(\mathbf{X}_{0}\right)$ includes the rigid body rotation and the homogeneous deformation of the subvolume $\mathrm{D}$. The 3 components of the displacement vector and the 9 components of the displacement gradient $\left(\partial \mathrm{u}_{\mathrm{i}} / \partial \mathrm{x}_{\mathrm{j}}\left(\mathbf{X}_{0}\right)\right)$ are considered as optimization parameters hereafter. The best parameters characterizing this approximation ( 3 for rigid body translation only or 12 scalars taking into account local deformation) are those minimizing a certain correlation coefficient $\mathrm{C}$ measuring the resemblance of gray level distributions in $\mathrm{D}$ and its transform. As in the two-dimensional case [19], can be written according to different formulations. We chose a dimensionless expression of scalar product type combined with a statistical formulation, which subtracts from each gray level its average on D:

$\mathrm{C}=1-\frac{\sum_{\mathbf{X} \in \mathrm{D}}\left[f(\mathbf{X})-\bar{f}_{D}\right]\left[g(\mathbf{x}(\mathbf{X}))-\bar{g}_{D}\right]}{{\sqrt{\sum_{\mathbf{X} \in \mathrm{D}}\left[f(\mathbf{X})-\bar{f}_{D}\right]}}^{2} \sum_{\mathbf{X} \in \mathrm{D}}\left[g(\mathbf{x}(\mathbf{X}))-\bar{g}_{D}\right]^{2}}$

$\mathrm{X}$ refers to the voxels of D. $f$ and $g$ designate respectively the gray levels of the reference and deformed images, with $\overline{\mathrm{f}}_{\mathrm{D}}$ and $\overline{\mathrm{g}}_{\mathrm{D}}$ their averages on D. This formulation is appropriate for images exhibiting contrast and brightness evolutions during in situ experiments, such as images of SMCT. The method is thoroughly described in Bornert et al. [23-28]. In the present work the software CMV3D [23-28] was used to quantify the local deformation in the reconstructed volumes scanned in situ at ID 15 . In the present application, only 3 translation components were optimized for each point of the regular 3D grid, owing to the overall low level of strain, below a few percent. The size of the correlation windows $\mathrm{D}$ was $30 \times 30 \times 30$ voxels $^{3}$, coinciding with the pitch of the virtual grid. Subvoxel accuracy can be reached for the evaluation of these components by means of a trilinear interpolation of the gray levels the deformed image, required for the computation of $\mathrm{g}(\mathbf{x}(\mathbf{X}))$ and its gradient in Eq. (4). Once this set of displacements is evaluated, they can be considered as the degrees of freedom of a 3D regular finite element mesh based on 8-noded elements with tri-linear shape function, generating a continuous displacement field throughout the analyzed region of interest. Average strains, either at the scale of each individual finite element or at the larger one of sets of elements defining some regions of interest, can then be calculated with standard FE integration routines. Detail of such procedures can be found in $[28,29]$. In particular, volume changes can be evaluated from the first invariant of these average strain tensors, as discussed below. It is noted that the accuracy of these strain measurements is proportional to the size of the averaging domains as the information used to optimize the displacement field increases with the domain volume. Hence, to optimize accuracy at constant spatial resolution, overlapping volumes could be used.

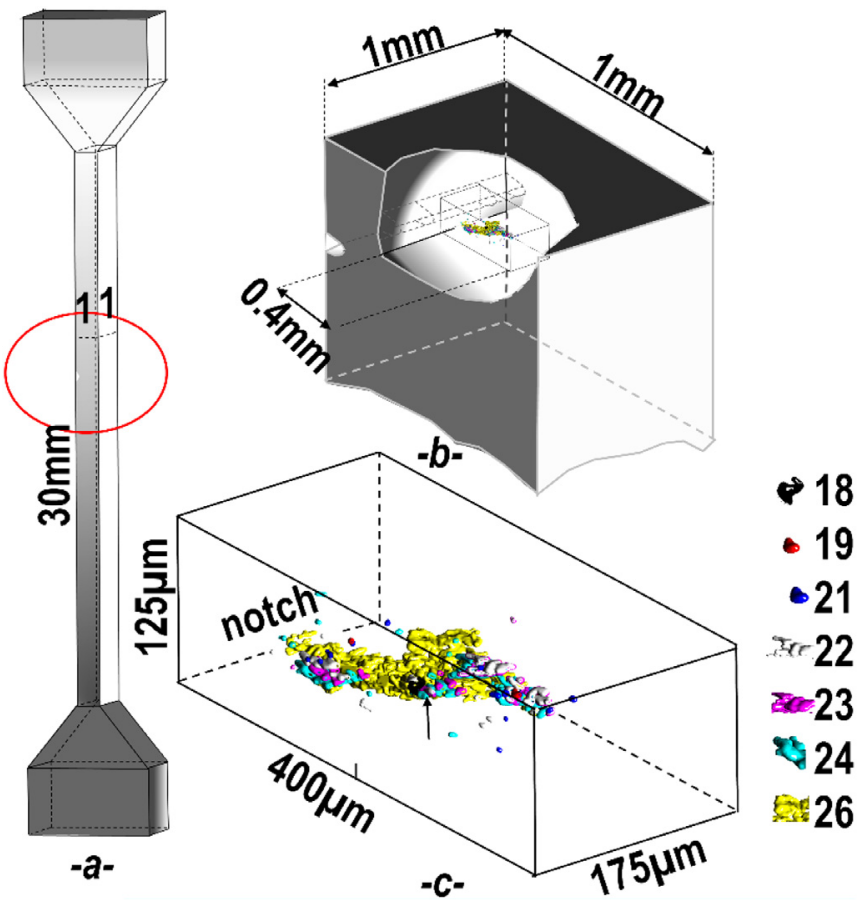

Fig. 3. (a) Tensile specimen geometry with the notch used for in situ observations at ID 15 ESRF, (b) Bounding box of the observed voids relative to the notch and (c) bounding box with the voids forming the final crack. Different colors represent the result of gray level segmentation at the different loading stages combined with a particle tracking algorithm.

\section{Results and discussion}

\subsection{Void coalescence and final crack formation}

After tomographic observations and image segmentation, a "particle"-tracking algorithm was applied to analyze void coalescence. Starting from the last deformation state prior to specimen failure, the tracking algorithm was used to remount in time the damage sequence by determining the voids leading to final crack formation. The result is shown in Fig. 3, where the voids are represented in specific colors corresponding to a particular state of the overall deformation. First, loading state 26 was analyzed, where voids have coalesced into a $0.4 \mathrm{~mm}$ long crack corresponding to almost half of the specimen width (shown in yellow). Then the tracking algorithm was used to analyze prior loading stages and the corresponding voids were segmented. Segmentation reveals significant presence of voids only after stage 18 when damage (voids) becomes concentrated into a $0.1 \mathrm{~mm}$ thin strip-like region due 


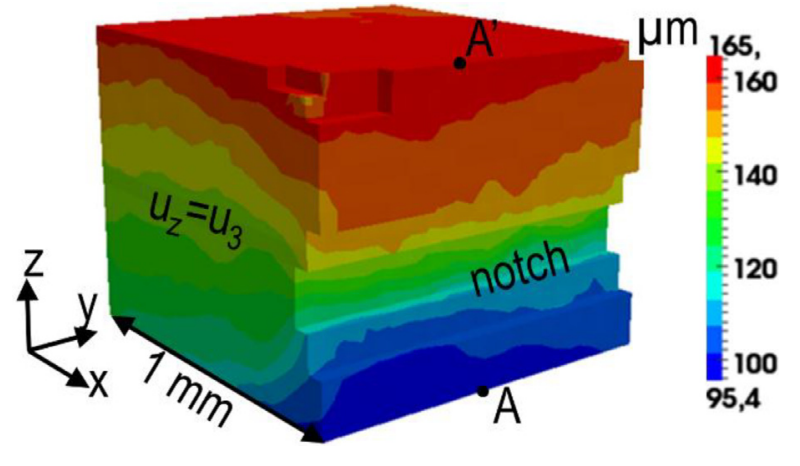

Fig. 4. Axial (tensile direction) component of the displacement vector determined by 3D image correlation (CMV3D) in a $30 \mathrm{~mm}$ long specimen submitted to an overall displacement of $370 \mu \mathrm{m}$.

to coalescence. Void coalescence (state 18 in black) starts "inside" the material some $200 \mu \mathrm{m}$ away from the notch. Up to the last state (26) new cavities "appear" outside the final crack. The most significant conclusion from this "visual" analysis is the following: Void coalescence is confined to a small layer of material with a height of about $\frac{1}{4}$ of the final crack length. We will now analyze damage generation prior to this final void coalescence and crack formation.

\subsection{Void volume fraction evolution during tensile testing}

After tomographic reconstruction and image segmentation, a void was counted only if it "contained" at least 3 adjacent voxels in each direction $(\mathrm{x}, \mathrm{y}, \mathrm{z})$, which represents a minimum void volume of 27 voxels or $(3 \times 1.4 \mu \mathrm{m})^{3}=72 \mu \mathrm{m}^{3}$. The corresponding linear size is of about $4.2 \mu \mathrm{m}$. To quantify the volume fraction of small voids the displacement field was determined by 3D image correlation in a volume of $1 \mathrm{~mm}^{3}$ centered on the notch. Hence, the analyzed material is more than 8 times higher and 5 times wider than the size of the final crack precursor (stage 26 in yellow). State zero was considered as reference. Image correlation was based on sub-volumes (D) of $30^{3}$ voxels. Fig. 4 shows the axial displacement field close to the notch at stage 24 corresponding to a macroscopic displacement of $370 \mu \mathrm{m}$ and hence an overall strain of about $1.2 \%$. The axial strain $\varepsilon_{\mathrm{zz}}$ in the observed volume is only slightly higher and the displacement $\mathrm{u}_{\mathrm{z}}$ is almost linear of $\mathrm{z}$. Thus, the notch allows easy observation, but does not alter too much the displacement and strain fields.

Neglecting the elastic deformation, which is very small compared to the displacements caused by damage and assuming that the $\alpha$-Al matrix is incompressible, every volume change detected by $3 \mathrm{D}$ image correlation is interpreted as void formation or growth. Then the following relation between the trace of the strain rate tensor $\dot{\mathrm{E}}_{\mathrm{kk}}$ and time derivative of the void volume fraction $\left(f=\mathrm{V}_{\text {voids }} / \mathrm{V}_{\text {total }}\right)$ holds:

$\dot{\mathrm{E}}_{\mathrm{kk}}=\frac{\dot{\mathrm{V}}}{\mathrm{V}}=\frac{\dot{\mathrm{f}}}{(1-\mathrm{f})}$,

which integrated over time leads to an explicit expression of the void volume fraction.

$\mathrm{f}=1-\left(1-\mathrm{f}_{0}\right) \exp \left(-\mathrm{E}_{\mathrm{kk}}\right)$.

$\mathrm{f}_{0}$ stands for the initial void volume fraction, which is considered negligible, so the void volume fraction at a given state only depends on the trace of the strain tensor.

$\mathrm{f} \approx 1-\exp \left(-\mathrm{E}_{\mathrm{kk}}\right) \approx \mathrm{E}_{\mathrm{kk}}$

Eq. (7) was applied to the entire volume analyzed by 3D image correlation to estimate the evolution of the mean void volume fraction. Fig. 5 shows the void volume fraction as a function of the applied strain. The void volume fraction prior to failure is about $1.4 \%$. On the same figure, the volume fraction of voids detected by simple gray value threshold (for voids larger than $4.2 \mu \mathrm{m}$ ) is also shown. The comparison of the

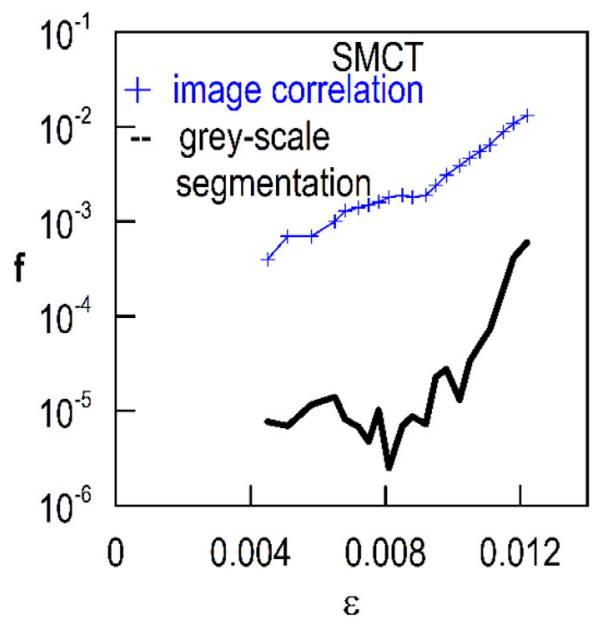

Fig. 5. Void volume fraction as a function of applied strain (a) 3D image correlation and segmentation, (b) Gray level segmentation.

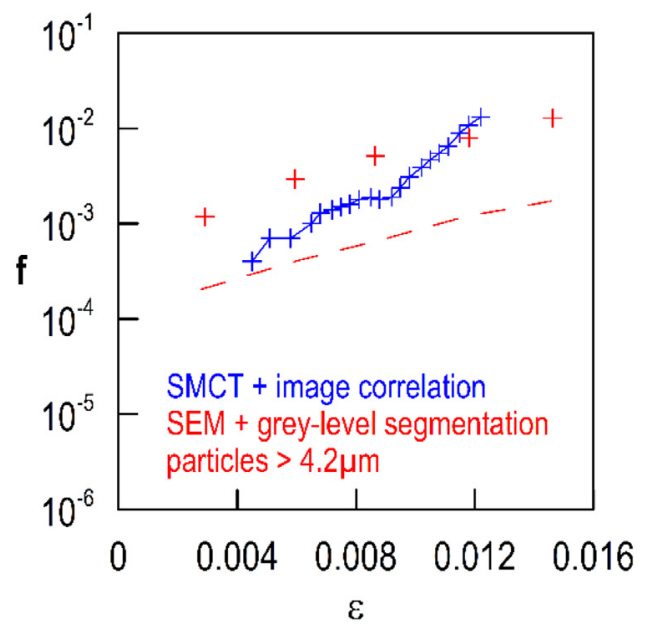

Fig. 6. Void surface fraction as a function of applied strain during the in situ tensile test in SEM (red crosses) and comparison with 3D measurements (blue). The dashed line in red shows the fraction of voids larger than $4.2 \mu \mathrm{m}$ obtained from SEM imaging.

two curves highlights that image correlation recovers a volume fraction that is of about one order of magnitude larger than usual segmentation, which suggests that most of the voids have a volume much smaller than the selected threshold. The surface fraction of voids was also determined during in situ tensile tests performed in the SEM over a surface of $\left(\sim 0.9 \times 1.2 \mathrm{~mm}^{2}\right)$ with a pixel size of $0.585 \mu \mathrm{m}$. The results shown in Fig. 6 match well with the values obtained from 3D image correlation. For comparison the surface fraction of voids larger than $4.2 \mu \mathrm{m}$ is also shown, which corresponds to the void volume fraction determined from SMCT reconstructions by gray level threshold segmentation. The difference between SMCT and SEM is attributed mainly to the larger area observed in 2D and much less sampling points in the 2D observations. Effectively, if the high resolution of the SEM presents a major advantage, during the large time needed for image acquisition, the sample recovers. Hence, these acquisitions were limited in number.

\section{Discussion}

\subsection{Relation between intermetallic particle break-up and void nucleation}

The previous results allow drawing some partial conclusions. Based on the lower spatial resolution of SMCT compared to SEM, one expects 


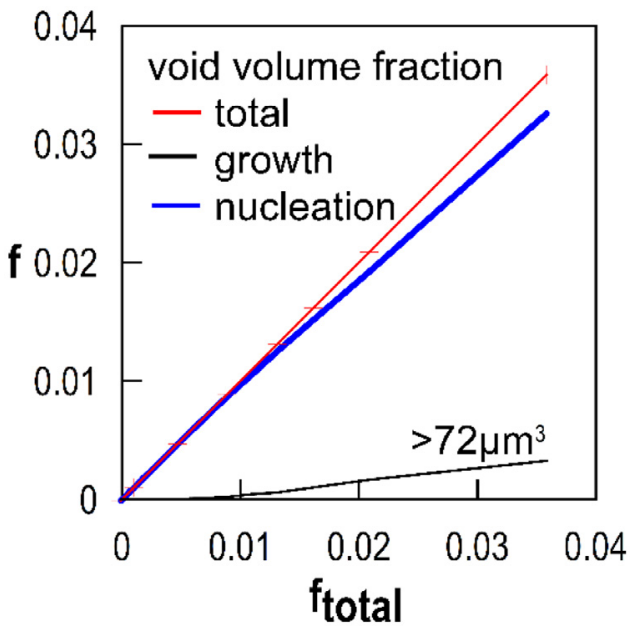

Fig. 7. Different contributions to the total void volume fraction determined by 3D image correlation.

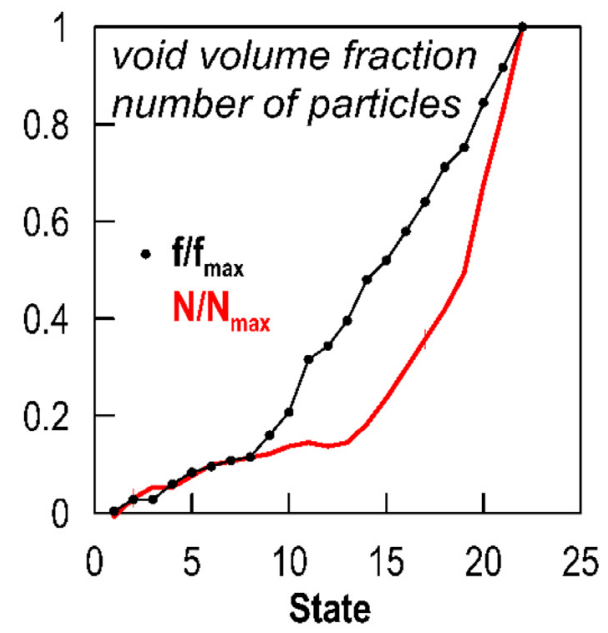

Fig. 8. Normalized void volume fraction and number of particles as function of the deformation state.

that the previous technique gives a much lower volume fraction of voids. This is really the case when classical segmentation methods based on gray level threshold are applied. However, applying 3D image correlation greatly improves the resolution of SMCT making it capable for detecting the global effect of small "invisible voids", which is a consequence of the subpixel resolution of image correlation techniques. SMCT combined with 3D image correlation gives a global void volume fraction in good agreement with SEM observations. Although the fraction of voids larger than $4.2 \mu \mathrm{m}$ is very small the total void volume fraction increases up to $\sim 1.4 \%$ prior to final failure. Hence, there should be a large number of small newly nucleated voids, as no significant void growth is observed. Fig. 7 shows the variation of the void volume fraction of different populations. The difference between the total void volume fraction and the fraction of larger voids is termed here "nucleation". These voids are the main contribution to the total volume fraction while not growing beyond the $72 \mu \mathrm{m}^{3}$ limit.

The previous scenario is only possible by continuously creating (nucleation) new voids, which do not grow significantly during deformation. At this point, one plausible cause for void formation seems to be particle cracking with nucleation of cavities at the endpoints of each new branch. Fig. 8 depicts the normalized variation of the void volume fraction and the number of particles as function of the deformation state. Effectively, both variables seem closely related. The previous assumption is illustrated in Fig. 9 showing tomographic results. The initial particle is drawn in blue, while the first broken parts (state 5) appear in black. The non-convex parts of the particle break at small remote strains. The small shift between the initial particle (in blue) and the broken parts (in black) is probably due to very small (unavoidable) rigid displacements of the sample at the very onset of the tensile test. Finding the broken parts despite this small shift highlights the stability of the particle-tracking algorithm developed in [27]. Fig. 9b shows the broken parts (detected by tomographic observation) and hints the possible void nucleation sites.

\subsection{Estimation of the average void size based of IM breakage}

The overall stress triaxiality during the tensile test is close to $1 / 3$. This relatively small value does not lead to void growth. At the instant of particle breaking the stress field around each particle fragment is, of course, very complex with possible stress concentrations and increased stress triaxiality. Hence, the scenario suggested consists in void nucleation at the border of each fragment followed by limited growth. Indeed, after breaking-off, the small fragments may turn slightly to minimize the resistance to the matrix flow. This corresponds to the small displacements seen by the particle tracking algorithm based also on image correlation. But, the small size of the fragments does not lead to a significant change in the remote stress field. This assumption naturally leads to a void diameter close to the mean thickness of the intermetallic branches, which cannot be segmented by simple threshold, but it can be detected by 3D image correlation. Therefore, based on the high correlation coefficient found above (according to Fig. 9), we consider the number of voids proportional to the number of particles, which multiplies due to break-up. This hypothesis allows estimating the mean volume of the average void $\left\langle\mathrm{V}_{\text {voids }}\right\rangle$ from the void volume fraction $\mathrm{f}$, measured by

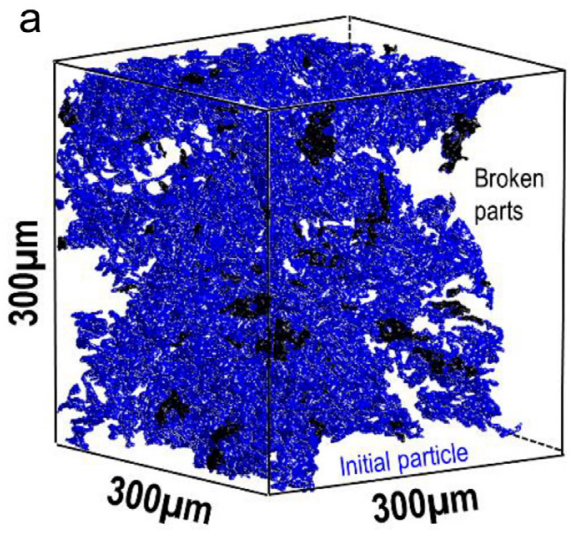

b

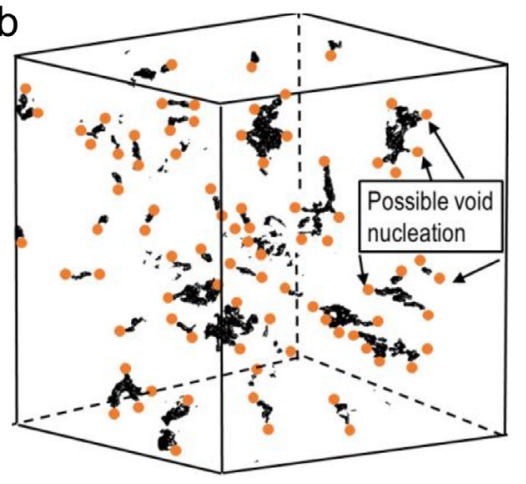

Fig. 9. Void nucleation scenario based on particle breakup. (a) Tomographic observation of IM particle break-up (in black) and (b) possible void nucleation sites. 


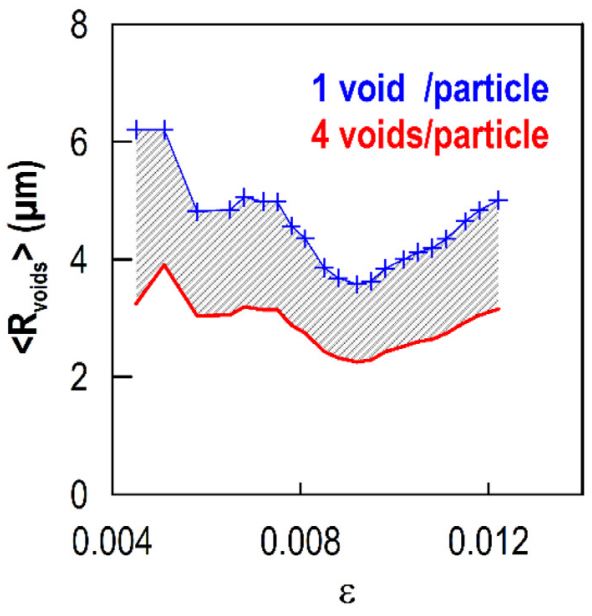

Fig. 10. Estimated mean particle radius based on 1 to 4 voids per particle fragment.

3D image correlation:

$\left\langle\mathrm{V}_{\text {voids }}\right\rangle=\mathrm{f} * \frac{\mathrm{V}_{\text {total }}}{\alpha \mathrm{N}_{\text {particles }}}$,

where $\alpha$ designates the number of voids per particle fragment. Assuming spherical voids the corresponding average radius was determined. The results considering either 1 or 4 voids nucleating at each new particle fragment are shown in Fig. 10. The predicted average radius lies between 4 and $6 \mu \mathrm{m}$ at the onset of damage and remains nearly constant during deformation (with a small decrease followed by a small increase).

An important outcome of the void nucleation hypotheses at the endpoints of broken branches and of their limited growth is the nearly constant value of the average void size (Fig. 10). If this hypothesis is correct, the average size should correlate with the average branch diameter. The latter was determined using an evaluation algorithm that considers the maximum sphere diameter that fits at a given location into the particle branch (Fig. 11a). By moving the sphere along the branch the local diameters were obtained and their histogram shown in Fig. 11b. Its mean value is of about 2.9 voxels $(\sim 4.1 \mu \mathrm{m})$ that corresponds to an average radius of $2 \mu \mathrm{m}$ and which is the lower limit of the estimation presented in Fig. 10. This means that the assumption of one void nucleation per branch rupture coupled to some limited growth seems to be a plausible scenario of damage progression in the studied material. Limited growth could be associated to a realignment of the branch with the material flow lines.

Fig. 12 shows the SEM image of a typical fracture surface. Voids and dimples characteristic of ductile failure may be observed. Less com-

a

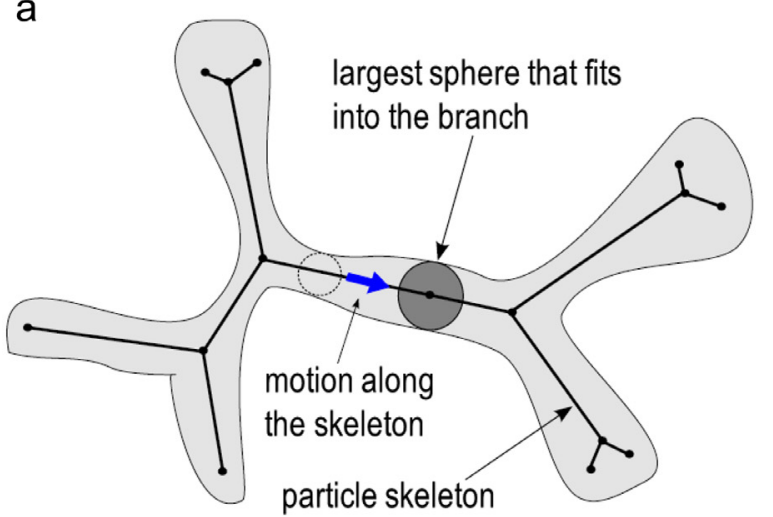

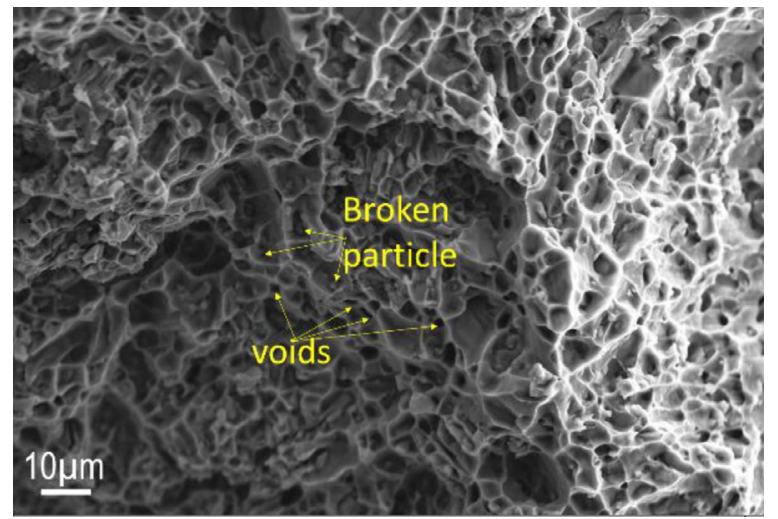

Fig. 12. SEM (secondary electron) image of the fracture surface of cast AlSi12Ni alloy after uniaxial tension.

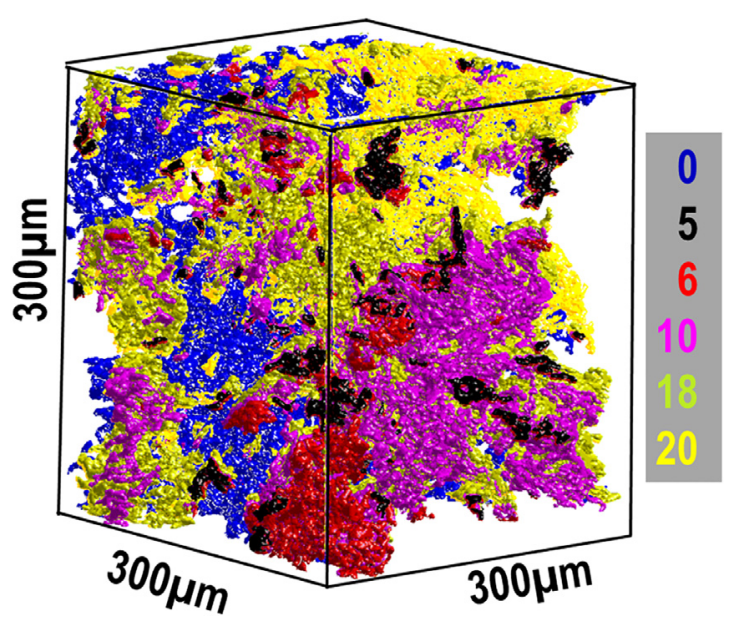

Fig. 13. Break-up sequence of an intermetallic particle obtained from SMCT reconstructions taken during in situ tensile testing at ID 15 ESRF.

mon is the quasi absence of secondary voids, while there is a considerable number of voids exhibiting a diameter between 2 and $4 \mu \mathrm{m}$. Some arrows point to broken intermetallic particles visible at few locations. Thus, voids are continuously "nucleated" at the border of broken particles and do essentially not grow. Intermetallic particles constitute a "casing" enclosing the aluminum matrix and limiting hereby potential void growth.

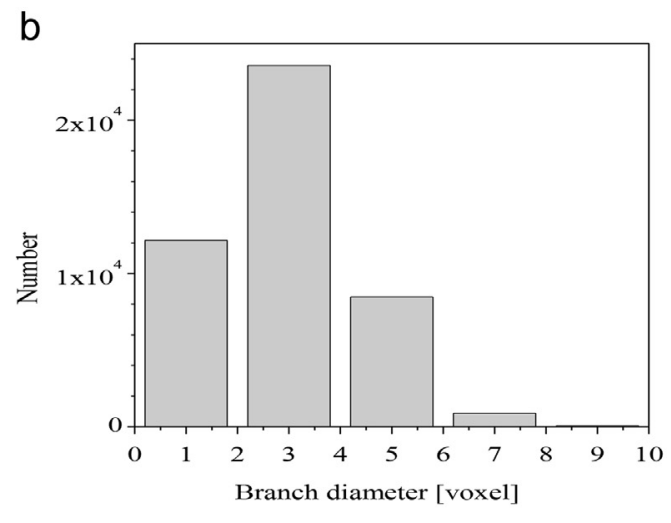

Fig. 11. a) Principle of the algorithm for branch diameter determination and b) the histogram of branch diameters. 

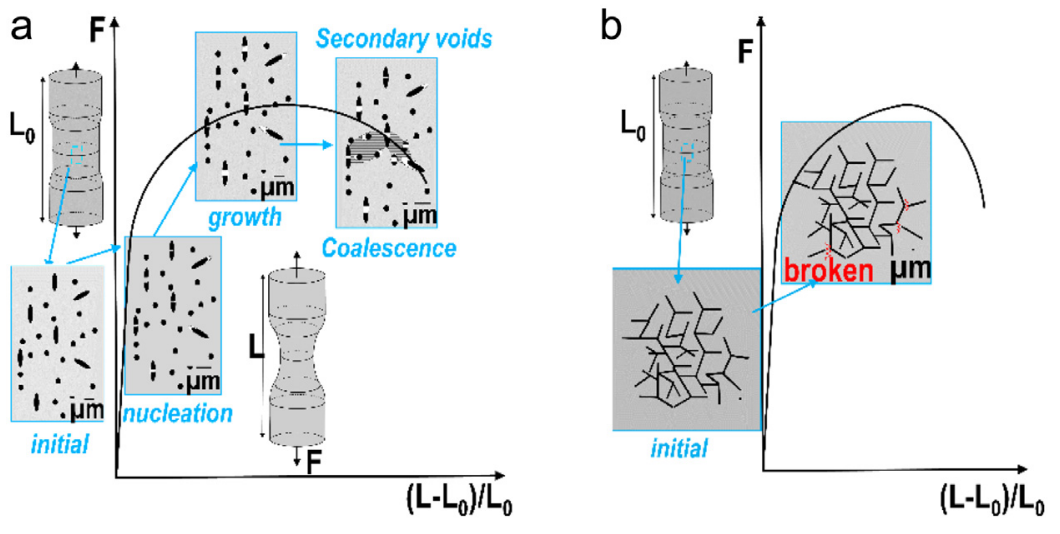

Fig. 14. Damage in a ductile matrix (a) known damage sequence in a matrix containing a dispersion of inclusions, (b) unknown damage sequence in a matrix containing a $3 \mathrm{D}$ connected network of particles.

\subsection{Differences in damage evolution between systems with disperse and interconnected second phases}

Present work explored damage development in an as cast AlSi12Ni alloy by a genuine combination of in situ observations at different length scales. In a previous paper, the microstructure was characterized thoroughly as an interconnected network of Si and intermetallic-particles. The microstructure of the considered AlSi12Ni alloy may be considered typical of most eutectic alloys. Besides in automotive applications, eutectic and pro-eutectic alloys regain a high interest as lead-free interconnects in micro-electronic devices and optimized microstructures obtained by additional fabrication methods. All these components are designed to exhibit ductile behavior under normal service conditions. Damage progression in the AlSi12Ni alloys is primarily determined by the breaking of the interconnected brittle IM phase as exemplified in Fig. 13 the sequence of consecutive SMCT reconstructions combined with a particle tracking algorithm [27]. The numbers correspond to the loading states when the corresponding broken parts (shown in different colors) were detected. The analysis leads naturally to the definition of synthetic microstructures reproducing typical "particle morphology", which becomes the major parameter influencing breakage. However, simulation of synthetic microstructures combined with finite element analyses implies long computer time and it is difficult to use as predictive tool for microstructure design. With this regard Moulin et al. $[30,31]$ presented an interesting first attempt to model break-up of nonconvex particles.

Summarizing, in materials with a disperse distribution of inclusions void nucleation, growth and coalescence are usually observed [9-12]. In materials exhibiting a network of interconnected particles only limited growth is observed, the network of particles constituting a casing that screens the overall stress field and hinders growth. Hence, the damage mechanism is completely different from the case of systems with disperse distribution of particles. Differences between the succession of damage in the two material types are summarized in Fig. 14.

This analysis clearly highlights continuous void nucleation during the complete strain history. The strong correlation between void volume fraction and number of broken particles indicates that almost all voids are nucleated at branches of broken particles. Other possible stress raisers than particles could be triple junctions. But, SEM images revealed no voids other than at broken particles.

\section{Conclusions}

Damage evolution during tensile straining of a cast AlSi12Ni alloy has been characterized by in situ SEM imaging and SMCT. Present work highlights continuous void nucleation by fragmentation of IM particles at the endpoints of broken branches. The growth of these voids is limited. Nevertheless, 3D image correlation detects the global effect of these small voids not captured by simple thresholding. Thus, these voids have a real effect on the strain field. The combination of SMCT and 3D image correlation allows a complete description of the void nucleation and coalescence induced by the fragmentation of intermetallic phases. The predictions of 3D image correlation were confirmed by and high resolution SEM imaging.

In ductile metals, containing a disperse distribution of inclusions (second phases) the commonly admitted damage sequence corresponds to nucleation, growth and coalescence with secondary void nucleation. This work highlights a completely different damage sequence in the presence of a network of interconnected phases. Effectively, void nucleation by particle breaking is observed, but void growth is limited. Hence, nucleation is continuous all over the strain path. The final crack formation is still controlled by void coalescence, but without secondary void formation.

\section{Declaration of Competing Interest}

The authors declare that they have no known competing financial interests or personal relationships that could have appeared to influence the work reported in this paper.

\section{Acknowledgement}

The authors acknowledge the European Synchrotron Radiation Facility for beam time in the framework of proposal no. MA560

\section{References}

[1] ASM technical report: Aluminum-Silicon Casting Alloys: Atlas of Microfractographs, 2004.

[2] L.F. Mondolfo, Aluminium Alloys: Structure and Properties, Butterworths, LondonBoston, 1976.

[3] J.R. Davis (Ed.), ASM Specialty Handbook: Aluminum and Aluminum Alloys, ASM International, 1993.

[4] S.Z. Lu, A. Hellawell, Modification of Al-Si alloys: microstructure, thermal analysis and mechanics, JOM 47 (2) (1995) 38-40.

[5] Z. Asghar, G. Requena, H.P. Degischer, P. Cloetens, Three-dimensional study of Ni aluminides in an AlSi12 alloy by means of light optical and synchrotron microtomography, Acta Mater. 57 (14) (2009) 4125-4132.

[6] Z. Asghar, G. Requena, F. Kubel, The role of ni and Fe aluminides on the elevated temperature strength of an AlSi12 alloy, Mater. Sci. Eng. A527 (21-22) (2010) 5691-5698.

[7] C.L. Chen, A. Richter, R.C. Thomson, Investigation of mechanical properties of intermetallic phases in multi-component Al-Si alloys using hot-stage nanoindentation, Intermetallics 18 (4) (2010) 499-508.

[8] G. Guillermo Requena, G. Garce's, Zahid Asghar, E. Esteban Marks, P. Staron, P. Cloetens, The effect of the connectivity of rigid phases on strength of AlSi alloys, Adv Eng Mater 13 (8) (2011) 673-684.

[9] F.A. McClintock, A criterion for ductile fracture by the growth of holes, J. Appl. Mech. 35 (35) (1968) 363-371.

[10] A.L. Gurson, Continuum theory of ductile rupture by void nucleation and growth. I. Yield criteria and flow rules for porous ductile media, J. Eng. Mater. Technol. 99 (1977) 2-15. 
[11] R.H. Van Stone, T.B. Cox, R. Low Jr, J.A. Psioda, Microstructural aspects of fracture by dimpled rupture, Int. Metals Rev. 30 (4) (1985) 157-180.

[12] G. Huber, Y. Brechet, T. Pardoen, Predictive model for void nucleation and void growth controlled ductility in quasi-eutectic cast aluminium alloys, Acta Mater 53 (2005) 2739-2749.

[13] K. Dzieciol, A. Borbély, F. Sket, A. Isaac, M. Di Michiel, P. Cloetens, T.h. Buslaps, A.R. Pyzalla, Void growth in copper during high-temperature power-law creep, Acta Mater. 59 (2) (2011) 671-677.

[14] E. Maire, P.J. Withers, Quantitative X-ray tomography, Int. Mater. Rev. 59 (1) (2014) $1-43$.

[15] A. Tireira, G. Requena, S. Sao Jao, A. Borbély, H. Klocker, Rupture of intermetallic networks and strain localization in cast AlSi12Ni alloy: 2D and 3D characterization, Acta Mater. 112 (2016) 162-170.

[16] J.Y. Buffiere, E. Maire, J. Adrien, J.P. Masse, E. Boller, In situ experiments with X-ray tomography: an attractive tool for experimental mechanics, Exp. Mech. 50 (3) (2010) 289-305.

[17] I. Kimura, T. Takamori, Image processing of flow around a circular cylinder using crosscorrelation technique, Flow Vis. IV (1986) 221-226.

[18] R. Adrian, Particle imaging techniques for experimental fluid mechanics, Ann. Rev. Fluid Mech. 23 (1991) 261-304.

[19] T. Chu, W. Ranson, M. Sutton, W. Peters, Applications of the digital-image-correlation techniques to experimental mechanics, Exp. Mech. 25 (3) (1985) 232-244.

[20] Z.L. Kahm-Jetter, T.C. Chu, Three-dimensional displacement measurements using digital image correlation and photogrammic analysis, Exp. Mech. 30 (1) (1990) 10-16.

[21] H. Bruck, S. McNeill, M. Sutton, W. Peters, Digital image correlation using Newton-Raphson method of partial differential correction, Exp. Mech. 29 (1) (1989) 261-267.
[22] D. Garcia, J.J. Orteu, L. Penazzi, A combined temporal tracking and stereo-correlation technique for accurate measurement of 3D displacements: application to sheet metal forming, J. Mater. Process. Technol. 125-126 (2002) 736-742.

[23] B.K. Bay, T.S. Smith, D.P. Fyhrie, M. Saad, Digital volume correlation: three-dimensional strain mapping using X-ray tomography, Exp. Mech. 39 (n3) (1999) 217-226.

[24] T.S. Smith, B.K. Bay, M.M. Rashid, Digital volume correlation including rotational degrees of freedom during minimization, Exp. Mech. 42 (n 3) (2002) 272-278.

[25] E. Verhulp, B. van Rietbergen, R. Huiskes, A three-dimensional digital correlation for strain measurements in microstructures, J. Biomech. 37 (9) (2004) 1313-1320.

[26] M. Bornert, P. Doumalin, Micromechanical applications of digital image correlation techniques, in: P. Jacquot, J. Fournier (Eds.), Interferometry in Speckle Light, Theory and Applications, Springer, 2000, pp. 67-74.

[27] M. Bornert, P. Doumalin, E. Soppa, et al., Computational and experimental investigations of the local strain field in elastoplastic two-phase materials, in: D. Miannay, et al. (Eds.), Advances in Mechanical behaviour, Plasticity and Damage, 1, EUROMAT, 2000, pp. 323-328.

[28] M. Bornert, O. Castelnau, J.M. Chaix, P. Doumalin, T. Fournel, D. Jeulin, E. Maire, M. Moreaud, H. Moulinec, Analyse tridimensionnelle expérimentale et théorique des champs locaux dans un composite non linéaire », colloque d'étape du programme matériaux, toulouse 2004 (CD-Rom), CNRS (2004).

[29] N. Lenoir, M. Bornert, J. Desrues, P. Bésuelle, G. Viggiani, Volumetric digital image correlation applied to X-Ray microtomography images form triaxial compression tests on argillaceaous rocks, Strain 43 (3) (2007) 193-205.

[30] N. Moulin, D. Jeulin, H. Klöcker, Stress concentrations in non-convex elastic particles embedded in a ductile matrix, Int. J. Eng. Sci. 47 (2) (2009) 170-191.

[31] N. Moulin, E. Parra-Denis, D. Jeulin, C.h. Ducottet, A. Bigot, E. Boller, E. Maire, C. Barat, H. Klöcker, Constituent particle break-up during hot rolling of AA 5182, Adv. Eng. Mater. 12 (1-2) (2010) 20-29. 